\title{
Existence-stability theorems for strong vector set-valued equilibrium problems in reflexive Banach spaces
}

\section{Gang Wang*}

\section{"Correspondence:}

wgglj1977@163.com

School of Management, Qufu

Normal University, Rizhao,

Shandong 276826, China

\section{空 Springer}

\begin{abstract}
In this paper, we develop existence and stability theorems for strong vector set-valued equilibrium problems in reflexive Banach spaces. Based on asymptotic cone theory, we present the equivalent characterizations on the nonemptiness and boundedness of the solution set for strong vector set-valued equilibrium problems. Furthermore, stability results are established for strong vector set-valued equilibrium problems, when both the mapping and the constraint set are perturbed by different parameters.
\end{abstract}

MSC: 49K30; 90C29

Keywords: strong vector set-valued equilibrium problems; nonemptiness and boundedness; stability analysis

\section{Introduction}

Let $X$ be a real reflexive Banach space with its dual space $X^{*}$ and $K \subseteq X$ be a closed set. Let $F: K \times K \rightarrow R$ be a real-valued bifunction. The equilibrium problems (for short EP) is to find $\bar{x} \in K$ such that

(EP) $\quad F(\bar{x}, y) \geq 0, \quad \forall y \in K$.

The equilibrium problems play an important role in economics, finance, image reconstruction, ecology, transportation, network, and so on (see, e.g., [1-4]). Later, many researchers extended (EP) to the vector set-valued case in different ways; see [5-7] and the references therein.

Let $F: K \times K \rightarrow 2^{Y}$ be a set-valued mapping, where $Y$ is a real normed space with an ordered cone $C$, that is, a pointed, closed, and convex cone. It is well known that weak vector set-valued equilibrium problems (for short WVSEP) include two basic types. The first type is to find $\bar{x} \in K$ such that

$$
\text { (FWVSEP) } \quad F(\bar{x}, y) \nsubseteq-\operatorname{int} C, \quad \forall y \in K .
$$

The second type is to find $\bar{x} \in K$ such that

$$
\text { (SWVSEP) } \quad F(\bar{x}, y) \cap-\operatorname{int} C=\emptyset, \quad \forall y \in K,
$$

where int $C$ denotes the interior of $C$.

(c) 2015 Wang. This article is distributed under the terms of the Creative Commons Attribution 4.0 International License (http://creativecommons.org/licenses/by/4.0/), which permits unrestricted use, distribution, and reproduction in any medium, provided you give appropriate credit to the original author(s) and the source, provide a link to the Creative Commons license, and indicate if changes were made. 
It is worth noting that strong vector set-valued equilibrium problems (for short SVSEP) include two basic types, too. The first type is to find $\bar{x} \in K$ such that

(FSVSEP) $\quad F(\bar{x}, y) \subseteq C, \quad \forall y \in K$.

The second type is to find $\bar{x} \in K$ such that

(SSVSEP) $\quad F(\bar{x}, y) \cap C \neq \emptyset, \quad \forall y \in K$.

The issues of nonemptiness and boundedness of the solution set are among the most interesting and important problems in the theory of (WVSEP), as they can guarantee the weak convergence of some solution algorithms $[8,9]$ in infinite dimensional spaces. For (FWVSEP), based on dual formulations, Ansari et al. [6, 7] proved the existence theorems under generalized pseudomonotonicity conditions. For (SWVSEP), several necessary and/or sufficient conditions for the solution set to be nonempty and bounded were established in $[10,11]$. Furthermore, the semicontinuity and connectedness of (approximate) solution sets can be found in [12-15] for weak vector set-valued equilibrium problems. On the other hand, if int $C=\emptyset$, then (WVSEP) cannot be studied. It is well known that for the classical Banach spaces $l^{p}, L_{p}$, where $1<p<+\infty$, the standard ordered cone has an empty interior [16]. Thus, for $C$-monotone-type (SVSEP), finding sufficient and/or necessary conditions for the nonemptiness and boundedness of the solution set is very important. To our knowledge, existence results proposed in [17] can be considered as a pioneering work for (SVSEP). Characterizations of nonemptiness and boundedness of the solution set for strong vector equilibrium problems were derived in different spaces [18, 19]. Recently, Long et al. [20] obtained the existence theorems for the generalized strong vector quasi-equilibrium problems by the Kakutani-Fan-Glicksberg fixed point theorem on compact sets. For (FSVSEP), on noncompact sets, Wang et al. [21] obtained some existence theorems by virtue of the Brouwer fixed point theorem in general real Hausdorff topological vector spaces. Since the characterizations of nonemptiness and boundedness of the solution set for strong vector equilibrium problems can be derived when $F$ is a single-valued map, it is natural to ask whether characterizations on nonemptiness and boundedness of the solution set for (SVSEP) can be obtained in the case that $F$ is multivalued, which constitutes the motivation of this article. In this paper, we present equivalent characterizations on the nonemptiness and boundedness of the solution set for (SVSEP) by means of the asymptotic cone theory in which the decision space is a real reflexive Banach space. Then we apply the equivalent characterizations to establish the stability theorems for (SVSEP) on a noncompact set, when both the mapping and the constraint set are perturbed by different parameters.

The rest of the paper is organized as follows. In Section 2, we introduce some basic notations and preliminary results. In Section 3, under suitable conditions we investigate the equivalence between the nonemptiness and boundedness of the solution set and the asymptotic cone $R_{2}=\{0\}$ for (SSVSEP). Stability results are presented for (SVSEP) on a noncompact set, when both the mapping and the constraint set are perturbed by different parameters in Section 4. Our results generalize and extend some results of $[3-7,13-15$, 19-21] in some sense. 


\section{Notations and preliminaries}

In this section, we introduce some basic notations and preliminary results.

Consider the following modeling: The first type strong vector set-valued equilibrium problems, abbreviated by (FSVSEP), is to find $\bar{x} \in K$ such that

(FSVSEP) $\quad F(\bar{x}, y) \subseteq C, \quad \forall y \in K$.

For (FSVSEP), its dual problem is to find $\bar{x} \in K$ such that

(DFSVSEP) $\quad F(y, \bar{x}) \subseteq-C, \quad \forall y \in K$.

We denote the solution set of (FSVSEP) and the solution set of (DFSVSEP) by $S S_{K}$ and $S S_{K}^{D}$, respectively.

The second type strong vector set-valued equilibrium problems, abbreviated by (SSVSEP), is to find $\bar{x} \in K$ such that

(SSVSEP) $\quad F(\bar{x}, y) \cap C \neq \emptyset, \quad \forall y \in K$.

For (SSVSEP), its dual problem is to find $\bar{x} \in K$ such that

(DSSVSEP) $\quad F(y, \bar{x}) \cap-C \neq \emptyset, \quad \forall y \in K$.

We denote the solution set of (SSVSEP) and the solution set of (DSSVSEP) by $S_{K}$ and $S_{K}^{D}$, respectively.

It is easy to see $S S_{K} \subseteq S_{K}$ and $S S_{K}^{D} \subseteq S_{K}^{D}$.

Definition 2.1 [22] Let $K$ be a nonempty, closed, and convex subset of a real reflexive Banach space $X$ with dual space $X^{*}$. The dual cone $K^{*}$ of $K$ is defined as

$$
K^{*}=\left\{x^{*} \in X^{*}:\left\langle x^{*}, x\right\rangle \geq 0, \forall x \in K\right\}
$$

It is well known that

$$
\operatorname{int} K^{*}=\left\{x^{*} \in X^{*}:\left\langle x^{*}, x\right\rangle>0, \forall x \in K \backslash\{0\}\right\},
$$

where 'int' means the interior of a set. The asymptotic cone $K^{\infty}$ and the barrier cone $\operatorname{barr}(K)$ of $K$ are, respectively, defined by

$$
K^{\infty}=\left\{d \in X \mid \exists t_{k} \rightarrow+\infty, \exists x_{k} \in K \text { with } \frac{x_{k}}{t_{k}} \rightarrow d\right\}
$$

and

$$
\operatorname{barr}(K)=\left\{x^{*} \in X^{*} \mid \sup _{x \in K}\left\langle x^{*}, x\right\rangle<+\infty\right\},
$$

where $\rightarrow$ stands for the weak convergence. 
The asymptotic cone $K^{\infty}$ has the following useful properties.

Lemma 2.1 [22] Let $K \subset X$ be nonempty and closed. Then the following conclusions hold:

(i) $K^{\infty}$ is a closed cone.

(ii) If $K$ is convex, then $K^{\infty}=\{d \in X \mid x+t d \in K, \forall t>0\}$, for all $x \in K$.

(iii) If $K$ is a convex cone, then $K^{\infty}=K$.

The following result can be found in Proposition 2.2 of [23] showing the property of $K$.

Lemma 2.2 Let $K$ be a nonempty, closed, and convex subset of a real reflexive Banach space $X$. If $\operatorname{barr}(K)$ has a nonempty interior, then there does not exist $\left\{x_{n}\right\} \subseteq K$ with $\left\|x_{n}\right\| \rightarrow$ $+\infty$ such that the origin is a weak limit of $\left\{\frac{x_{n}}{\left\|x_{n}\right\|}\right\}$. If $K$ is a cone, then there does not exist $\left\{d_{n}\right\} \subset K$ with each $\left\|d_{n}\right\|=1$ such that $d_{n} \rightarrow 0$.

To obtain the characterization of the solution sets for (SSVSEP) and (FSVSEP), we recall generalized monotonicity and generalized convexity [24].

Definition 2.2 [24] Let $K$ be a nonempty, closed, and convex subset of $X$. A mapping $F: K \times K \rightarrow 2^{Y}$ is said to be

(i) type I $C$-pseudomonotone if, for all $x, y \in K$,

$$
F(x, y) \subseteq C \quad \Rightarrow \quad F(y, x) \subseteq-C
$$

(ii) type II $C$-pseudomonotone if, for all $x, y \in K$,

$$
F(x, y) \cap C \neq \emptyset \Rightarrow F(y, x) \cap-C \neq \emptyset .
$$

It is easy to verify that type I $C$-pseudomonotonicity implies type II $C$-pseudomonotonicity. However, the converse is not true.

Example 2.1 Let $X=R^{1}, K=[1,+\infty), Y=R^{2}, C=R_{+}^{2}$. Let $F: K \times K \rightarrow 2^{Y}$ be defined by

$$
F(x, y)= \begin{cases}{[-|y-x|,|y-x|],} & \forall x, y \in[1,+\infty), \\ {[-1,1],} & \forall x, y \in[1,+\infty) .\end{cases}
$$

Clearly, its dual is

$$
F(y, x)= \begin{cases}{[-|x-y|,|x-y|],} & \forall x, y \in[1,+\infty), \\ {[-1,1],} & \forall x, y \in[1,+\infty) .\end{cases}
$$

For all $x, y \in K$, we have

$$
F(x, y) \cap C \neq \emptyset \quad \Rightarrow \quad F(y, x) \cap-C \neq \emptyset .
$$

However, we cannot find $x, y \in K$ such that

$$
F(x, y) \subseteq C .
$$

Thus, $F$ is not type I $C$-pseudomonotone. 
Definition 2.3 [24] The mapping $F: K \times K \rightarrow 2^{Y}$ is said to be strongly

(i) type I $C$-diagonally quasi-convex in the first argument if, for any finite $\left\{x_{1}, x_{2}, \ldots, x_{n}\right\} \subseteq K$ and any $t_{i} \geq 0$ with $\sum_{i=1}^{n} t_{i}=1, x=\sum_{i=1}^{n} t_{i} x_{i}$, and for some $x_{i} \in K$, one has

$$
F\left(x_{i}, x\right) \subseteq-C
$$

(ii) type II $C$-diagonally quasi-convex in the first argument if, for any finite $\left\{x_{1}, x_{2}, \ldots, x_{n}\right\} \subseteq K$ and any $t_{i} \geq 0$ with $\sum_{i=1}^{n} t_{i}=1, x=\sum_{i=1}^{n} t_{i} x_{i}$, and for some $x_{i} \in K$, one has

$$
F\left(x_{i}, x\right) \cap-C \neq \emptyset
$$

(iii) $C$-convex in the second argument if, for any $y_{1}, y_{2} \in K$ and $t \in(0,1)$, one has

$$
t F\left(x, y_{1}\right)+(1-t) F\left(x, y_{2}\right) \subseteq F\left(x, t y_{1}+(1-t) y_{2}\right)+C .
$$

The following lemma is the well-known KKM theorem, we refer the reader to Lemma 1 of [25].

Lemma 2.3 [25] Let $K \subseteq X$ be a nonempty convex of a topological vector space $X$ and $F: K \rightarrow 2^{X}$ be a set-valued mapping from $K$ into $X$ satisfying the following properties:

(i) $F$ is a KKM mapping: For every finite subset $A$ of $E, \operatorname{co}(A) \subseteq \bigcup_{x \in A} F(x)$, where $\operatorname{co}(\cdot)$ stands for the convex hull;

(ii) $F(x)$ is closed in $X$ for every $x \in K$;

(iii) $F\left(x_{0}\right)$ is compact in $X$ for some $x_{0} \in K$.

Then $\bigcap_{x \in K} F(x) \neq \emptyset$.

\section{The nonemptiness and boundedness of the solution sets for (FSVSEP) and (SSVSEP)}

In this section, we present the equivalent characterizations of the solution set for strong vector set-valued equilibrium problems to be nonempty and bounded based on asymptotic cone theory.

Theorem 3.1 Let $K$ be a nonempty, closed, and convex subset of $X$ with $\operatorname{int}(\operatorname{barr}(K)) \neq \emptyset$. Suppose that $F: K \times K \rightarrow 2^{Y}$ satisfies the following:

(i) $F$ is type II C-pseudomonotone and $F(x, x) \subset C \cap-C, \forall x \in K$;

(ii) the set $\{x \in K: F(x, y) \cap C \neq \emptyset\}$ is closed for any $y \in K$ and $F$ is strongly type II $C$-diagonally quasi-convex in the first argument;

(iii) the set $\{y \in K: F(x, y) \cap-C \neq \emptyset\}$ is closed for any $x \in K$ and $F$ is $C$-convex in the second argument.

Then the following statements are equivalent:

(I) (SSVSEP) has a nonempty, convex, closed, and bounded solution set;

(II) (DSSVSEP) has a nonempty, convex, closed, and bounded solution set;

(III) $R_{2}=\left\{d \in K_{\infty}: F(y, y+t d) \cap-C \neq \emptyset, \forall y \in K, t>0\right\}=\{0\}$; 
(IV) there exists a bounded set $D \subset K$ such that for every $x \in K \backslash D$, there exists some $y \in D$ such that

$$
F(y, x) \cap-C=\emptyset
$$

Proof (I) $\Leftrightarrow$ (II). Suppose that (SSVSEP) has a nonempty, convex, closed, and bounded solution set. By the type II $C$-pseudomonotonity of $F$, we obtain $S_{K} \subseteq S_{K}^{D}$.

On the other hand, let us verify $S_{K}^{D} \subseteq S_{K}$. Take any $x^{*} \in S_{K}^{D}$, obviously

$$
F\left(y, x^{*}\right) \cap-C \neq \emptyset
$$

For every $y \in K$, consider $x_{t}=x^{*}+t\left(y-x^{*}\right), \forall t \in(0,1)$. Clearly, $x_{t} \in K$. The $C$-convexity of $F\left(x_{t}, \cdot\right)$ implies that

$$
(1-t) F\left(x_{t}, x^{*}\right)+t F\left(x_{t}, y\right) \subseteq F\left(x_{t}, x_{t}\right)+C \subseteq C+C \subseteq C
$$

Let us by contradiction show $t F\left(x_{t}, y\right) \cap C \neq \emptyset$. Suppose the contrary, then $t F\left(x_{t}, y\right) \subseteq Y \backslash C$. Consequently,

$$
(1-t) F\left(x_{t}, x^{*}\right) \subseteq Y \backslash-C+C \subseteq Y \backslash-C,
$$

which contradicts (3.1). Noting that $Y \backslash C$ is a cone, we deduce

$$
F\left(x_{t}, y\right) \cap C \neq \emptyset \text {. }
$$

By assumption (ii), letting $t \rightarrow 0$ in (3.3), one has

$$
F\left(x^{*}, y\right) \cap C \neq \emptyset, \quad \forall y \in K \text {. }
$$

Hence, (SSVSEP) has a nonempty, convex, closed, and bounded solution set if and only if (DSSVSEP) has a nonempty, convex, closed, and bounded solution set.

$$
\text { (II) } \Rightarrow \text { (III). We know }
$$

$$
S_{K}^{D}=\bigcap_{y \in K}\{x \in K: F(y, x) \cap-C \neq \emptyset\} .
$$

For fixed $y \in K$, one has

$$
\begin{aligned}
& S_{0}(y)=\{x \in K: F(y, x) \cap-C \neq \emptyset\}, \\
& S_{K}^{D}=\bigcap_{y \in K} K \cap S_{0}(y) .
\end{aligned}
$$

Since (DSSVSEP) has a nonempty, convex, closed, and bounded solution set, we have

$$
\left(S_{K}^{D}\right)^{\infty}=\left(\bigcap_{y \in K} K \cap S_{0}(y)\right)^{\infty}=\bigcap_{y \in K}\left(K \cap S_{0}(y)\right)^{\infty}=\bigcap_{y \in K} K^{\infty} \cap S_{0}(y)^{\infty}
$$


On the other hand, one has

$$
\begin{aligned}
\{d & \left.\in K^{\infty}: F(y, y+t d) \cap-C \neq \emptyset, \forall y \in K, t>0\right\} \\
& =\bigcap_{y \in K}\left\{d \in K^{\infty}: F(y, y+t d) \cap-C \neq \emptyset, \forall t>0\right\} \\
& =\bigcap_{y \in K}\left\{d \in K^{\infty}: y+t d \in S_{0}(y), \forall t>0\right\} \\
& =\bigcap_{y \in K}\left\{d \in K^{\infty}: d \in S_{0}(y)^{\infty}\right\}=\bigcap_{y \in K} K^{\infty} \cap S_{0}(y)^{\infty} .
\end{aligned}
$$

Thus, (III) holds.

(III) $\Rightarrow$ (IV). If (III) does not hold, then there exists a sequence $\left\{x_{n}\right\} \subset K$ such that $n<$ $\left\|x_{n}\right\|$ with

$$
F\left(y, x_{n}\right) \cap-C \neq \emptyset,
$$

for $y \in K$ with $\|y\| \leq n$. For fixed $y \in K$ and $t>0$, without loss of generality, we may take a subsequence $\left\{x_{n_{k}}\right\}$ of $\left\{x_{n}\right\}$ such that

$$
\frac{t}{\left\|x_{n_{k}}-y\right\|} \in(0,1), \quad \lim _{k \rightarrow+\infty} \frac{x_{n_{k}}-y}{\left\|x_{n_{k}}-y\right\|}=\lim _{k \rightarrow+\infty} \frac{x_{n_{k}}}{\left\|x_{n_{k}}\right\|} \rightarrow d_{0} \in K_{\infty} .
$$

Noting that $X$ is a reflexive Banach space and $\operatorname{int}(\operatorname{barr}(K)) \neq \emptyset$, from Lemma 2.2 , we have $d_{0} \neq 0$. The $C$-convexity of $F(x, \cdot)$ implies

$$
\left(1-\frac{t}{\left\|\left(x_{n_{k}}-y\right)\right\|}\right) F(y, y)+\frac{t}{\left\|\left(x_{n_{k}}-y\right)\right\|} F\left(y, x_{n_{k}}\right) \subseteq F\left(y, y+\frac{t\left(x_{n_{k}}-y\right)}{\left\|\left(x_{n_{k}}-y\right)\right\|}\right)+C .
$$

From $F(y, y) \subseteq C$ and $F\left(y, x_{n_{k}}\right) \cap-C \neq \emptyset$, one has

$$
F\left(y, y+\frac{t\left(x_{n_{k}}-y\right)}{\left\|\left(x_{n_{k}}-y\right)\right\|}\right) \cap-C \neq \emptyset
$$

By assumption (iii), letting $t \rightarrow 0$ in (3.4), we obtain

$$
F\left(y, y+t d_{0}\right) \cap-C \neq \emptyset
$$

which contradicts (III). So (IV) holds.

$$
(\mathrm{IV}) \Rightarrow(\mathrm{II}) \text {. Set } M: K \times K \rightarrow 2^{K} \text { by }
$$

$$
M(y)=\{x \in K: F(y, x) \cap-C \neq \varnothing\}, \quad \forall y \in K .
$$

It is easy to verify that $M(y)$ is a closed subset of $K$. We shall show that $M$ is a KKM mapping. We may assume that $D$ is a bounded, closed, and convex set (otherwise, consider the closed convex hull of $D$ instead of $D$ ). Let $\left\{y_{1}, \ldots, y_{m}\right\}$ be finite number of points in $K$ and let $L=\operatorname{co}\left(D \cup\left\{y_{1}, \ldots, y_{m}\right\}\right)$. So, $L$ is a weakly compact convex set, since $X$ is a reflexive Banach space. Consider the set-valued mapping $\overline{M(y)}$ defined by

$$
\overline{M(y)}=\{x \in L: F(y, x) \cap-C \neq \emptyset\}, \quad \forall y \in L .
$$


Obviously, $\overline{M(y)}$ is a weakly compact convex subset of $L$. We claim $\overline{M(y)}$ has the finite intersection property.

Indeed, for all $\left\{x_{1}, x_{2} \ldots, x_{p}\right\} \subset L, \sum_{t_{i}}^{p}=1, t_{i} \geq 0$, and $\bar{x}=\sum_{i=1}^{p} t_{i} x_{i}$, it follows from assumption (ii) that there exists $x_{i} \in L$ such that

$$
F\left(x_{i}, \bar{x}\right) \cap-C \neq \emptyset,
$$

namely, $\bar{x} \in \bigcup_{i=1}^{p} \overline{M\left(x_{i}\right)}$. Hence, $\overline{M(y)}$ has the finite intersection property. Noting that $\overline{M(y)}$ has the finite intersection property and $\overline{M(y)}$ is a weakly compact convex subset, we obtain, from Lemma 2.3

$$
\emptyset \neq \bigcap_{y \in L} \overline{M(y)}
$$

Let $\bar{x} \in \bigcap_{y \in L} \overline{M(y)}$, we have

$$
F(y, \bar{x}) \cap-C \neq \emptyset, \quad \forall y \in L .
$$

We assert that $\bar{x} \in D$. Suppose to the contrary that if there exists some $\bar{x} \in \overline{M(y)}$ but $\bar{x} \notin D$, by the assumption (IV), one has

$$
F(y, \bar{x}) \cap-C=\emptyset, \quad \text { for some } y \in D .
$$

Consequently, $\bar{x} \notin \overline{M(y)}$, which is a contradiction with (3.6). Thus,

$$
\emptyset \neq \bigcap_{y \in L} \overline{M(y)} \subset D .
$$

For $\bar{x} \in \bigcap_{y \in L} \overline{M(y)}$, by (3.7), we deduce $\bar{x} \in \bigcap_{i=1}^{m}\left(M\left(y_{i}\right) \cap D\right)$, which implies the collection $\{M(y) \cap D: y \in K\}$ has the finite intersection property. Since for each $y \in K,(M(y) \cap D)$ is weakly compact, it follows from Lemma 2.3 that $\bigcap_{y \in K}(M(y) \cap D) \neq \emptyset$, which coincides with the solution set of (DSSVSEP). Notice that

$$
S_{K}^{D}=\bigcap_{y \in K}\{x \in K: F(y, x) \cap-C \neq \emptyset\} .
$$

From assumption (iii), it is easy to see that $S_{K}^{D}$ is closed. Next, we shall show that $S_{K}^{D}$ is convex. For all $x_{1}, x_{2} \in S_{K}^{D}$ and $t \in[0,1]$, from the $C$-convexity of $F(y, \cdot)$, we have

$$
t F\left(y, x_{1}\right)+(1-t) F\left(y, x_{2}\right) \subseteq F\left(y, t x_{1}+(1-t) x_{2}\right)+C .
$$

It is easy to verify $F\left(y, t x_{1}+(1-t) x_{2}\right) \cap-C \neq \emptyset$. Thus, (DSSVSEP) has a nonempty, convex, closed, and bounded solution set.

Remark 3.1 When $F$ is a single-valued and $C=R_{+}^{1}$, a similar result to (III) can be obtained in [3] for equilibrium problems and in [26] for the variational inequality. Furthermore, if $D$ is closed, convex, and bounded, the result (IV) was proved in [4] for equilibrium problems. It is worth to stress that Theorem 3.1 is a new result for (SSVSEP), since similar results cannot be found in $[18,19]$. 
Now by the following example we illustrate Theorem 3.1.

Example 3.1 Let $X=R^{1}, K=[0,+\infty), Y=R^{2}, C=R_{+}^{2}$. Let $F: K \times K \rightarrow 2^{Y}$ be defined by

$$
F(x, y)= \begin{cases}{[-(y-x), y-x],} & \forall x, y \in[0,+\infty) \\ \langle x, y-x\rangle, & \forall x, y \in[0,+\infty)\end{cases}
$$

Clearly, its dual problem is

$$
F(y, x)= \begin{cases}{[-(x-y),(x-y)],} & \forall x, y \in[0,+\infty), \\ \langle y, x-y\rangle, & \forall x, y \in[0,+\infty)\end{cases}
$$

It is easy to see that assumptions (i)-(iii) are satisfied. We can verify that (I) $\Leftrightarrow$ (II), that is, $S_{K}=S_{K}^{D}=\{0\}$; (III) holds, namely, $R_{2}=\{0\}$; and if we take the bounded set $\{0\}=D \subset K$, then (IV) is true.

Similar to the proof of Theorem 3.1, the following result holds for (FSVSEP).

Theorem 3.2 Let $K$ be a nonempty, closed, and convex subset of $X$ with int $\operatorname{barr}(K) \neq \emptyset$. Suppose that $F: K \times K \rightarrow 2^{Y}$ satisfies the following:

(i) $F$ is type I C-pseudomonotone and $F(x, x) \subset C \cap-C, \forall x \in K$;

(ii) the set $\{x \in K: F(x, y) \subseteq C\}$ is closed for any $y \in K$ and $F$ is strongly type I $C$-diagonally quasi-convex in the first argument;

(iii) the set $\{y \in K: F(x, y) \subseteq-C\}$ is closed for any $x \in K$ and $F$ is $C$-convex in the second argument.

Then the following statements are equivalent:

(I) (FSVSEP) has a nonempty, convex, closed, and bounded solution set;

(II) (DFSVSEP) has a nonempty, convex, closed, and bounded solution set;

(III) $R_{1}=\left\{d \in K_{\infty}: F(y, y+t d) \subseteq-C, \forall y \in K, t>0\right\}=\{0\}$;

(IV) there exists a bounded set $D \subset K$ such that for every $x \in K \backslash D$, there exists some $y \in D$ such that

$$
F(y, x) \nsubseteq-C
$$

\section{Stability analysis for (FSVSEP) and (SSVSEP)}

In this section, we shall establish the stability theorems for (FSVSEP) and (SSVSEP) when the mapping $F$ and the domain set $K$ are simultaneously perturbed by different parameters. We take (SSVSEP) and (DSSVSEP) as examples to present the stability theorems.

First recall some important notions and results. Let $\left(Z_{1}, d_{1}\right)$ and $\left(Z_{2}, d_{2}\right)$ be two metric spaces. Let $K(p)$ be perturbed by a parameter $p$, which varies over $\left(Z_{1}, d_{1}\right)$, that is, $K: Z_{1} \rightarrow$ $2^{X}$ is a set-valued mapping with nonempty, closed, and convex values. Let $F$ be perturbed by a parameter $z$, which varies over $\left(Z_{2}, d_{2}\right)$, that is, $F: Z_{2} \times K \times K \rightarrow 2^{Y}$ is a parametric set-valued mapping.

Consider the perturbed second type strong vector set-valued equilibrium problems, denoted by (PSSVSEP), which consists in finding $\bar{x} \in K(p)$ such that

(PSSVSEP) $\quad F(z, \bar{x}, y) \cap C \neq \emptyset, \quad \forall y \in K(p)$. 
Its dual problem is to find $\bar{x} \in K(p)$ such that

(PDSSVSEP) $\quad F(z, y, \bar{x}) \cap-C \neq \emptyset, \quad \forall y \in K(p)$.

We denote the solution set of (PSSVSEP) and solution set of (PDSSVSEP) by $S_{K(p)}(z)$ and $S_{K(p)}^{D}(z)$, respectively.

Definition 4.1 Let $X$ and $Y$ be topological spaces. A set-valued mapping $T: X \rightarrow 2^{Y}$ is said to be

(i) upper semicontinuous at $x_{0} \in X$ if and if only, for any neighborhood $U\left(T\left(x_{0}\right)\right)$ of $T\left(x_{0}\right)$, there exists a neighborhood $U\left(x_{0}\right)$ of $x_{0}$ such that

$$
T(x) \subset U\left(T\left(x_{0}\right)\right), \quad \forall x \in U\left(x_{0}\right)
$$

(ii) lower semicontinuous at $x_{0} \in X$ if and if only, for any $y_{0} \in T\left(x_{0}\right)$ and any neighborhood $U\left(y_{0}\right)$ of $y_{0}$, there exists a neighborhood $U\left(x_{0}\right)$ of $x_{0}$ such that

$$
T(x) \cap U\left(y_{0}\right) \neq \emptyset, \quad \forall x \in U\left(x_{0}\right)
$$

(iii) continuous at $x_{0}$ if and if only it is both upper and lower semicontinuous at $x_{0}$.

It is evident that $T$ is lower semicontinuous at $x_{0} \in Y$ if and if only, for any net $\left\{x_{\beta}\right\}$ with $x_{\beta} \rightarrow x_{0}$ and $y_{0} \in T\left(x_{0}\right)$, there exists a net $\left\{y_{\beta}\right\}$ with $y_{\beta} \in T\left(x_{\beta}\right)$ such that $y_{\beta} \rightarrow y_{0}$.

The following lemma will be useful for proving our results.

Lemma 4.1 [26] Let $(Z, d)$ be a metric space and $u_{0} \in Z$ be a given point. Let $L: Z \rightarrow 2^{X}$ be a set-valued mapping with nonempty values and be upper semicontinuous at $u_{0}$. Then there exists a neighborhood $U$ of $u_{0}$ such that $L(u)^{\infty} \subset L\left(u_{0}\right)^{\infty}$ for all $u \in U$.

Theorem 4.1 Let $\left(Z_{1}, d_{1}\right),\left(Z_{2}, d_{2}\right)$ be metric spaces and let $K: Z_{1} \rightarrow 2^{X}$ be a set-valued mapping with nonempty, closed, and convex values. Suppose that

(i) $K(p)$ is continuous at $p_{0}$ and $\operatorname{int}(\operatorname{barr} K(p)) \neq \emptyset, \forall p \in Z_{1}$;

(ii) $F(z, x, x) \subseteq C \cap-C, \forall x \in K(p), z \in Z_{2}$;

(iii) the set $\left\{(z, y) \in\left(Z_{2}, K(p)\right): F(z, x, y) \cap-C \neq \emptyset\right\}$ is closed for any $x \in K(p)$; $F$ is

$C$-convex in the third argument for any $x \in K(p)$ and $z \in Z_{2}$, and $F$ is strongly type II $C$-diagonally quasi-convex in the second argument for any $z \in Z_{2}, y \in K(p)$;

(iv) $S_{K\left(p_{0}\right)}^{D}\left(z_{0}\right)$ is nonempty and bounded.

Then

(I) there exists a neighborhood $U_{1} \times U_{2}$ of $\left(p_{0}, z_{0}\right)$ such that (PDSSVSEP) has a nonempty and bounded solution set for all $(p, z) \in\left(U_{1}, U_{2}\right)$;

(II) $\lim \sup _{(p, z) \rightarrow\left(p_{0}, z_{0}\right)} S_{K(p)}^{D}(z) \subseteq S_{K\left(p_{0}\right)}^{D}\left(z_{0}\right)$.

Proof Since $S_{K\left(p_{0}\right)}^{D}\left(z_{0}\right)$ is nonempty and bounded, from Theorem 3.1, we deduce

$$
R_{2}\left(p_{0}\right)=\bigcap_{y \in K\left(p_{0}\right)}\left\{d \in K\left(p_{0}\right)_{\infty}: F\left(z_{0}, y, y+t d\right) \cap-C \neq \emptyset, t>0\right\}=\{0\} .
$$


We claim that there exists a neighborhood $\left(U_{1}, U_{2}\right)$ of $\left(p_{0}, z_{0}\right)$ such that

$$
R_{2}(p)=\bigcap_{y \in K(p)}\left\{d \in K(p)_{\infty}: F(z, y, y+t d) \cap-C \neq \emptyset, t>0\right\}=\{0\} .
$$

Assume by contradiction that there exists $\left(p_{n}, z_{n}\right) \rightarrow\left(p_{0}, z_{0}\right)$ such that $R_{2}\left(p_{n}\right) \neq\{0\}$. Noting that $K$ is lower semicontinuous at $p_{0}$, for any $y \in K\left(p_{0}\right)$, we have $y_{n} \in K\left(p_{n}\right)$ such that $y_{n} \rightarrow y$. Together with $z_{n} \rightarrow z$, we have $\left(y_{n}, z_{n}\right) \rightarrow\left(y, z_{0}\right)$. Thus, we can select a sequence $\left\{d_{n}\right\}$ such that

$$
\bigcap_{y \in K\left(p_{n}\right)}\left\{d_{n} \in K\left(p_{n}\right)_{\infty}: F\left(z_{n}, y_{n}, y_{n}+t d_{n}\right) \cap-C \neq \emptyset, t>0\right\}
$$

with $\left\|d_{n}\right\|=1$ for all $n=1,2, \ldots, n$. As $X$ is reflexive, without loss of generality, we can assume that $d_{n} \rightarrow d_{0}$. It follows from Lemma 2.2 that $d_{0} \neq 0$. We claim that $d_{0} \in K\left(p_{0}\right)^{\infty}$. Since $K$ is upper semicontinuous at $p_{0}$ and $d_{n} \in K\left(p_{n}\right)^{\infty}$, from Lemma 4.1, one has $d_{n} \in K\left(p_{0}\right)^{\infty}$, for all sufficiently large $n$. By the closure of $K\left(p_{0}\right)^{\infty}$, we have $d_{0} \in K\left(p_{0}\right)^{\infty}$. Noticing assumption (iii), taking the limit in (4.2), we have

$$
F\left(z_{0}, y, y+t d_{0}\right) \cap-C \neq \emptyset,
$$

which contradicts (4.1). Thus, the result (I) holds.

For the result (II), we need to prove that for any $(p, z) \rightarrow\left(p_{0}, z_{0}\right)$,

$$
\limsup _{(p, z) \rightarrow\left(p_{0}, z_{0}\right)} S_{K(p)}^{D}(z) \subseteq S_{K\left(p_{0}\right)}^{D}\left(z_{0}\right)
$$

Let $x \in \lim \sup _{(p, z) \rightarrow\left(p_{0}, z_{0}\right)} S_{K(p)}^{D}(z)$. Then there exists a sequence $x_{n_{k}} \in S_{K\left(p_{n_{k}}\right)}^{D}\left(z_{n_{k}}\right)$ such that $x_{n_{k}} \rightarrow x$ as $k \in \infty$. Since $K$ is upper semicontinuous at $p_{0}$, we obtain

$$
K\left(p_{n}\right) \subset K\left(p_{0}\right)+\frac{1}{n} B, \quad \text { for all sufficiently large } n,
$$

where $B$ denotes the closed unit ball. This together with $x_{n_{k}} \in K\left(p_{n_{k}}\right)$ implies that

$$
d\left(x_{n_{k}}, K\left(p_{0}\right)\right) \leq \frac{1}{n_{k}} \rightarrow 0 .
$$

Since $x_{n_{k}} \rightarrow x$ and $K\left(p_{0}\right)$ is closed and convex, one has $x \in K\left(p_{0}\right)$.

For any $y \in K\left(p_{0}\right)$, it follows from the lower semicontinuity of $K$ at $p_{0}$ that there exists $y_{n} \in K\left(p_{n}\right)$ with $y_{n} \rightarrow y$. By the assumption $x_{n_{k}} \in S_{K\left(p_{n_{k}}\right)}^{D}\left(z_{n_{k}}\right)$, we have

$$
F\left(z_{n}, y_{n}, x_{n}\right) \cap-C \neq \emptyset .
$$

From assumption (iii), taking the limit in (4.3), one has

$$
F\left(z_{0}, y, x\right) \cap-C \neq \emptyset .
$$

This yields $x \in S_{K\left(p_{0}\right)}^{D}\left(z_{0}\right)$. 


\section{Remark 4.1}

(i) In Theorem 4.1, $F$ is vector set-valued mapping, including the scalar variational inequality and scalar equilibrium problems. Thus, Theorem 4.1 generalizes and extends Theorem 4.1 of [23], Theorem 3.2 of [26], and Theorem 4.1 of [27] in some sense.

(ii) We establish the stability theorem for (DSSVSEP) in Theorem 4.1, when the mapping $F$ and the domain set $K$ are simultaneously perturbed by different parameters. Theorem 4.1 of [23] and Theorem 4.1 of [27] only show that the mapping $F$ or the domain set $K$ is perturbed, respectively.

As a consequence of Theorem 3.1 and Theorem 4.1, the following results follow immediately.

Corollary 4.1 Let $\left(Z_{1}, d_{1}\right),\left(Z_{2}, d_{2}\right)$ be metric spaces and let $K: Z_{1} \rightarrow 2^{X}$ be a set-valued mapping with nonempty, closed, and convex values. Suppose that

(i) $K(p)$ is continuous at $p_{0}$ and $\operatorname{int}(\operatorname{barr} K(p)) \neq \emptyset, \forall p \in Z_{1}$;

(ii) $F(z, x, x) \subseteq C \cap-C, \forall x \in K(p), z \in Z_{2}$;

(iii) the set $\left\{(z, x) \in\left(Z_{2}, K(p)\right): F(z, x, y) \cap-C \neq \emptyset\right\}$ is closed for any $y \in K(p) ; F(z, \cdot, \cdot)$ is type II $C$-pseudomonotone for any $z \in Z_{2}$;

(iv) the set $\left\{(z, y) \in\left(Z_{2}, K(p)\right): F(z, x, y) \cap-C \neq \emptyset\right\}$ is closed for any $x \in K(p) ; F$ is $C$-convex in the third argument for any $x \in K(p)$ and $z \in Z_{2}$, and $F$ is strongly type II $C$-diagonally quasi-convex in the second argument for any $z \in Z_{2}, y \in K(p)$;

(v) $S_{K\left(p_{0}\right)}\left(z_{0}\right)$ is nonempty and bounded.

Then

(I) there exists a neighborhood $U_{1} \times U_{2}$ of $\left(p_{0}, z_{0}\right)$ such that (PSSVSEP) has a nonempty and bounded solution set for all $(p, z) \in\left(U_{1}, U_{2}\right)$;

(II) $\lim \sup _{(p, z) \rightarrow\left(p_{0}, z_{0}\right)} S_{K(p)}(z) \subseteq S_{K\left(p_{0}\right)}\left(z_{0}\right)$.

Consider the perturbed the first type strong vector set-valued equilibrium problems, denoted by (PFSVSEP), which consists in finding $\bar{x} \in K(p)$ such that

(PFSVSEP) $\quad F(z, \bar{x}, y) \subseteq C, \quad \forall y \in K(p)$.

Its dual problem is to find $\bar{x} \in K(p)$ such that

(PDFSVSEP) $\quad F(z, y, \bar{x}) \subseteq-C, \quad \forall y \in K(p)$.

We denote the solution set of (PFSVSEP) and the solution set of (PDFSVSEP) by $S S_{K(p)}(z)$ and $S S_{K(p)}^{D}(z)$, respectively.

Similar to the proof of Theorem 4.1, we have the following stability results for (FSVSEP) and (DFSVSEP).

Theorem 4.2 Let $\left(Z_{1}, d_{1}\right),\left(Z_{2}, d_{2}\right)$ be metric spaces and let $K: Z_{1} \rightarrow 2^{X}$ be a set-valued mapping with nonempty, closed, and convex values. Suppose that

(i) $K(p)$ is continuous at $p_{0}$ and $\operatorname{int}(\operatorname{barr} K(p)) \neq \emptyset, \forall p \in Z_{1}$;

(ii) $F(z, x, x) \subseteq C \cap-C, \forall x \in K(p), z \in Z_{2}$; 
(iii) the set $\left\{(z, y) \in\left(Z_{2}, K(p)\right): F(z, x, y) \subseteq-C\right\}$ is closed for any $x \in K(p)$; $F$ is $C$-convex in the third argument for any $x \in K(p), z \in Z_{2}$, and $F$ is strongly type I $C$-diagonally quasi-convex in the second argument for any $z \in Z_{2}, y \in K(p)$;

(iv) $S S_{K\left(p_{0}\right)}^{D}\left(z_{0}\right)$ is nonempty and bounded.

Then

(I) there exists a neighborhood $U_{1} \times U_{2}$ of $\left(p_{0}, z_{0}\right)$ such that (PDFSVSEP) has a nonempty and bounded solution set for all $(p, z) \in\left(U_{1}, U_{2}\right)$;

(II) $\lim \sup _{(p, z) \rightarrow\left(p_{0}, z_{0}\right)} S S_{K(p)}^{D}(z) \subseteq S S_{K\left(p_{0}\right)}^{D}\left(z_{0}\right)$.

As a consequence of Theorem 3.2 and Theorem 4.2, the following results follow immediately.

Corollary 4.2 Let $\left(Z_{1}, d_{1}\right),\left(Z_{2}, d_{2}\right)$ be metric spaces and let $K: Z_{1} \rightarrow 2^{X}$ be a set-valued mapping with nonempty, closed, and convex values. Suppose that

(i) $K(p)$ is continuous at $p_{0}$ and $\operatorname{int}(\operatorname{barr} K(p)) \neq \emptyset, \forall p \in Z_{1}$;

(ii) $F(z, x, x) \subseteq C \cap-C, \forall x \in K(p), z \in Z_{2}$;

(iii) the set $\left\{(z, x) \in\left(Z_{2}, K(p)\right): F(z, x, y) \subseteq-C\right\}$ is closed for any $y \in K(p) ; F(z, \cdot, \cdot)$ is type I $C$-pseudomonotone for any $z \in Z_{2}$;

(iv) the set $\left\{(z, y) \in\left(Z_{2}, K(p)\right): F(z, x, y) \subseteq-C\right\}$ is closed for any $x \in K(p)$; $F$ is $C$-convex in the third argument for any $x \in K(p), z \in Z_{2}$, and $F$ is strongly type I $C$-diagonally quasi-convex in the second argument for any $z \in Z_{2}, y \in K(p)$;

(v) $S_{K\left(p_{0}\right)}\left(z_{0}\right)$ is nonempty and bounded.

Then

(I) there exists a neighborhood $U_{1} \times U_{2}$ of $\left(p_{0}, z_{0}\right)$ such that (PFSVSEP) has a nonempty and bounded solution set for all $(p, z) \in\left(U_{1}, U_{2}\right)$;

(II) $\lim \sup _{(p, z) \rightarrow\left(p_{0}, z_{0}\right)} S_{K(p)}(z) \subseteq S_{K\left(p_{0}\right)}\left(z_{0}\right)$.

\section{Competing interests}

The author declares that they have no competing interests.

\section{Acknowledgements}

This research is supported by the Specialized Research Fund for the Doctoral Program of Higher Education of China (20113705110002, 20113705120004), China Postdoctoral Science Foundation funded project (2013M531566) and Promotive Research Fund for Young and Middle-aged Scientists of Shandong Province (BS2012SF008), the Natural Science Foundation of China $(61403228,11171180,11401438)$. The author would like to thank the reviewers for their careful reading, insightful comments, and constructive suggestions, which helped improve the presentation of the paper.

Received: 5 May 2015 Accepted: 11 July 2015 Published online: 30 July 2015

\section{References}

1. Blum, E, Oettli, W: From optimization and variational inequalities to equilibrium problems. Math. Stud. 63, 123-145 (1994)

2. Bigi, G, Castellani, M, Pappalardo, M, Passacantando, M: Existence and solution methods for equilibria. Eur. J. Oper. Res. 227, 1-11 (2012)

3. Babazan, FF: Existence theorems for generalized noncoercive equilibrium problems: the quasiconvex case. SIAM J. Optim. 11, 675-690 (2000)

4. Bianchi, M, Pini, R: Coercivity conditions for equilibrium problems. J. Optim. Theory Appl. 124, 79-92 (2005)

5. Konnov, IV, Yao, JC: Existence of solutions for generalized vector equilibrium problems. J. Math. Anal. Appl. 233, 328-335 (1999)

6. Ansari, QH, Konnov, IV, Yao, JC: On generalized vector equilibrium problems. Nonlinear Anal. 47, $543-554$ (2001)

7. Ansari, QH, Siddiqi, AH, Wu, SY: Existence and duality of generalized vector equilibrium problems. J. Math. Anal. Appl. 259, 115-126 (2001)

8. Iusem, AN, Sosa, W: On the proximal point method for equilibrium problems in Hilbert spaces. Optimization 59 1259-1274 (2010)

9. Konnov, IV, Ali, MS: Descent methods for monotone equilibrium problems in Banach spaces. J. Comput. Appl. Math. $188,165-179(2006)$ 
10. Ansari, QH, Flores-Bazán, F: Recession methods for generalized vector equilibrium problems. J. Math. Anal. Appl. 321, 132-146 (2006)

11. Farajzadeh, AP, Harandi, AA: On the generalized vector equilibrium problems. J. Math. Anal. Appl. 344, 999-1004 (2008)

12. Peng, $Z Y$, Zhao, $Y$, Yang, $X M$ : Semicontinuity of approximate solution mappings to parametric set-valued weak vector equilibrium problems. Numer. Funct. Anal. Optim. 36(4), 481-500 (2015)

13. Peng, $Z Y$, Yang, $X M$, Peng, JW: On the lower semicontinuity of the solution mappings to parametric weak generalized Ky Fan inequality. J. Optim. Theory Appl. 152, 256-264 (2012)

14. Long, XJ, Peng, JW: Connectedness and compactness of weak efficient solutions for vector equilibrium problems. Bull. Korean Math. Soc. 48, 1225-1233 (2011)

15. Long, XJ, Huang, YQ, Peng, ZY: Optimality conditions for the Henig efficient solution of vector equilibrium problems with constraints. Optim. Lett. 5, 717-728 (2011)

16. Jahn, J: Mathematical Vector Optimization in Partially Ordered Linear Spaces. Peter Lang, Frankfurt (1986)

17. Ansari, QH, Oettli, W, Schlager, D: A generalization of vectorial equilibria. Math. Methods Oper. Res. 46, 147-152 (1997)

18. Huang, LG: Existence of solutions of vector equilibrium problems. Acta. Math. Sin. Chin. Ser. 52(5), 904-910 (2009)

19. Ansari, QH, Farajzadeh, AP, Schaible, S: Existence of solutions of strong vector equilibrium problems. Taiwan. J. Math. 16(1), 165-178 (2012)

20. Long, XJ, Huang, NJ, Teo, KL: Existence and stability of solutions for generalized strong vector quasi-equilibrium problem. Math. Comput. Model. 47, 445-451 (2008)

21. Wang, SH, Li, QY, Fu, JY: Strong vector equilibrium problems on noncompact sets. Bull. Malays. Math. Sci. Soc. 35(1), 119-132 (2012)

22. Auslender, A, Teboulle, M: Asymptotic Cones and Functions in Optimization and Variational Inequalities. Springer, Berlin (2003)

23. He, YR: Stable pseudomonotone variational inequality in reflexive Banach spaces. J. Math. Anal. Appl. 330, 352-363 (2007)

24. Chen, GY, Huang, XX, Yang, XQ: Vector Optimization: Set-Valued and Variational Analysis. Springer, Berlin (2005)

25. Fan, K: Some properties of sets related to fixed point theorems. Math. Ann. 266, 519-537 (1984)

26. Fan, JH, Zhong, RY: Stability analysis for variational inequality in reflexive Banach spaces. Nonlinear Anal. 69 , 2566-2574 (2008)

27. Hu, R, Fang, YP: Strict feasibility and stable solvability of bifunction variational inequalities. Nonlinear Anal. 75, $331-340(2012)$

\section{Submit your manuscript to a SpringerOpen ${ }^{\ominus}$ journal and benefit from:}

- Convenient online submission

Rigorous peer review

- Immediate publication on acceptance

- Open access: articles freely available online

- High visibility within the field

- Retaining the copyright to your article 